OPEN ACCESS

Edited by:

Jianguo Su,

Huazhong Agricultural

University, China

Reviewed by:

Darius Widera,

University of Reading,

United Kingdom

Johannes F. W. Greiner.

Bielefeld University, Germany

*Correspondence:

Tianjun Xu

tianjunxu@163.com

Specialty section: This article was submitted to

Comparative Immunology,

a section of the journal

Frontiers in Immunology

Received: 30 January 2018 Accepted: 09 April 2018

Published: 26 April 2018

Citation:

Zhao X, Huo R, Yan X and Xu T (2018) IRF3 Negatively Regulates

Toll-Like Receptor-Mediated NF- $\kappa B$

Signaling by Targeting TRIF for

Degradation in Teleost Fish.

Front. Immunol. 9:867.

doi: 10.3389/fimmu.2018.00867

\section{IRF3 Negatively Regulates Toll-Like Receptor-Mediated NF-кB Signaling by Targeting TRIF for Degradation in Teleost Fish}

\author{
Xueyan Zhao ${ }^{1,2}$, Ruixuan Huo ${ }^{2}$, Xiaolong Yan $^{2}$ and Tianjun $X^{1,2,3,4 *}$ \\ ${ }^{1}$ Key Laboratory of Exploration and Utilization of Aquatic Genetic Resources, Shanghai Ocean University, Ministry of \\ Education, Shanghai, China, ${ }^{2}$ College of Marine Science, Zhejiang Ocean University, Zhoushan, China, ${ }^{3}$ International \\ Research Center for Marine Biosciences at Shanghai Ocean University, Ministry of Science and Technology, Shanghai, \\ China, ${ }^{4}$ National Pathogen Collection Center for Aquatic Animals, Shanghai Ocean University, Shanghai, China
}

NF-кB signaling is tightly regulated and essential to innate and adaptive immune responses, its regulatory mechanism remains unclear in various organisms, especially teleosts. In this study, we reported that IRF3 can negatively regulate TRIF-mediated NF- $\mathrm{KB}$ signaling pathway. Overexpression of IRF3 can inhibit TRIF-mediated NF-kB signaling pathway. However, knockdown of IRF3 had an opposite effect. IRF3 can promote the degradation of TRIF protein in mammal and fish cells, but this effect could be inhibited by MG132 treatment. Furthermore, we found that the inhibitory effect of IRF3 primary depended on its IRF association domain domain. IRF3 is crucial for the polyubiquitination and proteasomal degradation of TRIF. Our findings indicate that IRF3 negatively regulates TLR-mediated NF-kB signaling pathway by targeting TRIF for ubiquitination and degradation. This study provides a novel evidence on the negative regulation of innate immune signaling pathways in teleost fish and thus might provide new insights into the regulatory mechanisms in mammals.

\section{Keywords: IRF3, TRIF, NF-кB, negative regulation, ubiquitination}

\section{INTRODUCTION}

Vertebrates have evolved various immune defense systems to protect themselves against invading microorganisms and eliminate infective pathogens (1). Innate and acquired immunity composed of two branches of the immune system. The innate immune system is the first line of host defense against pathogens, such as viruses and bacteria, and can recognize a limited, but highly conserved set of molecular structures known as pathogen-associated molecular patterns (PAMPs) (1). PAMPs are recognized by several classes of pattern-recognition receptors, such as toll-like receptors (TLRs), RIG-I-like receptors (RLRs), NOD-like receptors and C-type lectin-like receptors (2-5). The specific ligands are recognized by different receptors, and the ligand-receptor binding results in the activation of common downstream pathways, such as NF- $\mathrm{KB}, \mathrm{MAPK}$, and type I interferon, which further induce the expression of cytokines and chemokine genes that facilitate the clearing of pathogens (6). However, aberrant immune responses occur, which leads to severe or even fatal bacterial sepsis, autoimmune, and chronic inflammatory diseases $(7,8)$. Therefore, these crucial signaling pathways have to be tightly regulated to maintain immune balance, which is essential to both innate and adaptive immunity. 
Host cells can recognize viral products through PRRs, especially TLRs and RLRs, on the surfaces of the cytomembranes or in the cytoplasm during virus infection, and then the downstream IFNs or other relative cytokine production processes are triggerred through signal transduction, thereby establishing the antiviral state of a host (9-11). However, the excessive immune response often leads to many inflammatory and autoimmune diseases. Thus, a variety of regulatory factors are needed to tightly regulate the TLR or RLR signaling pathway to maintain immune balance. For example, NLRX1 inhibits the activity of RLH and interferon- $\beta$ promoter, which is mediated by MAVS (12). NLRX1 can inhibit TLR-induced NF- $\kappa \mathrm{B}$ signaling by interacting with TRAF6 or binding directly to an IKK complex; it also inhibits the phosphorylation of IKK, further facilitating the inhibition of NF- $\mathrm{\kappa B}$ activation and releasing of the proinflammatory cytokines (6). The COX5B physically interacts with MAVS and negatively regulates the MAVS-mediated antiviral pathway (13). In addition, the ubiquitin E3 ligase RAUL can negatively regulate type I interferon through the ubiquitination of transcription factors, such as IRF7 and IRF3 (14).

Toll-like receptors are type I transmembrane proteins and contain extracellular ectodomains with leucine-rich repeats, which are transmembrane domain; and an intracellular toll interleukin (IL)-1 receptor domain for the recruitment of downstream adapter proteins. To date, five of adaptor proteins have been identified, including MyD88, Myd88 adaptor-like, TRIF, TRAM, and SARM (15-17). Thus, the signaling pathways are divided into the MyD88-dependent and TRIF-dependent pathway. Recruited MyD88 proteins promote the phosphorylation of IRAKs and subsequently activate the expression of TRAF6, then the NF- $\kappa \mathrm{B}$ and MAPK pathways are activated and proinflammatory cytokines are induced (18-20). TLR3 and TLR4 can recruit of TRIF and subsequently to activate the TRIF-dependent pathway, then TRIF interacts with TBK1 to activate downstream IRF3 and NF- $\kappa B$, consequently TLR3 and TLR4 inducing signaling pathway can induce the producing of type I IFN and proinflammatory cytokines (18-20). To avoid harmful and inappropriate in inflammatory responses, a variety of mechanisms can negatively regulate the TLR signaling pathways (16). Furthermore, a variety of cases that regulated TRIF-mediated signaling pathway have been found. For instance, ADAM15 can negatively regulate TRIF-mediated NF- $\kappa B$ and IFN $\beta$ reporter gene activity (21). TRIM38 can negatively regulate the TLR3-mediated type I interferon signaling pathway by targeting TRIF for degradation (22). WWP2, an E3 ligase, can negatively regulate TLR3-mediated innate immune response by targeting TRIF for ubiquitination and degradation (23). To date, many instances that IRFs regulate MyD88-mediated signaling have been confirmed. Such as, IRF4, which competes with IRF5 for interacting with MyD88, acts as a negative regulator of TLR signaling (24). IRF5 interacts with MyD88 and TRAF6 and then activates cytokine gene transcription (25). However, there are very few reports that IRF family member can regulate TRIF-mediated $\mathrm{NF}-\kappa \mathrm{B}$ pathway signaling.

Although many genes in mammals are involved in the regulation of TLR signaling pathways, the mechanisms involved the regulation of these pathways are rarely reported in fish. Miiuy croaker (Miichthys miiuy) is an economically important marine fish. The study of this species have been conducted in-depth from transcriptome and whole-genome (26), to immune genes $(27,28)$, which left miiuy croaker as an excellent model for studying the mechanisms of some molecules in regulation of immune response of fish (29-31). In this study, we confirmed that IRF3 negatively regulated TRIF-mediated NF- $\kappa \mathrm{B}$ signaling pathway by targeting TRIF for degradation in teleosts. Overexpression of IRF3 can inhibit TRIF-mediated NF- $\mathrm{B}$ signaling pathway, whereas knockdown of IRF3 has an opposite effect. Fundamentally, IRF3 promotes TRIF degradation via the ubiquitin-proteasome pathway. In addition, we found that the IRF association domain (IAD) of IRF3 is crucial for TRIF proteasomal degradation. Furthermore, we found that IRF3 may inhibit TRIF expression and consequently result in decreasing the expression of downstream inflammatory cytokines. This study not only provides an evidence for the regulatory mechanism of TRIF signaling by IRFs and enriches the content of TLR signaling pathway in teleosts, but also might provide new insights into the regulatory mechanism in mammals.

\section{MATERIALS AND METHODS}

\section{Plasmids Construction}

To construct the expression plasmids, TRIF of the miiuy croaker was cloned into the Kpn I and BamH I sites of pEGFP-N1 and to the Kpn I and EcoR I sites of pCDNA3.1 (Invitrogen) with Flag tag; IRF3 of the miiuy croaker was cloned into the Kpn I and Xba I sites of pCDNA3.1 with myc tag; TBK1 of the miiuy croaker was cloned into the Hind III and EcoR I sites of pCDNA3.1 with HA tag; STAT1a of the miiuy croaker was cloned into the BamH I and EcoR I sites of pCDNA3.1 with myc tag; p65 of the miiuy croaker was cloned into the Hind III and Kpn I sites of pCDNA3.1 with Flag tag. The TAK1 expression plasmids were conducted using a ClonExpress II One Step Cloning Kit (Vazyme) with specific primers then was cloned into the pcDNA3.1. And the series of deletion mutants of IRF3, including IRF3 $\triangle$ DNA binding domain $(\Delta \mathrm{DBD}), \mathrm{IRF} 3 \Delta \mathrm{IAD}(\Delta \mathrm{IAD})$, and IRF3 $\Delta \mathrm{SRD}(\Delta \mathrm{SRD})$, were generated by PCR based on the IRF3 recombinant plasmid by using specific primers. The pRK5-HA-Ubiquitin-WT (Ubiquitin-HA) plasmid was purchased from Addgene. And the oligonucleotide of IRF3-shRNAs were designed and ligated into BamH I and EcoR I of pSIREN-RetroQZsGreen 1 vector (Clontech). The recombinant plasmid was confirmed by DNA sequencing. All primers were listed in Table 1. All of the plasmids were extracted using EndotoxinFree Plasmid DNA Miniprep Kit (Tiangen).

\section{Cell Culture and Transient Transfections}

Miiuy croaker macrophages were aseptically isolated from the head kidney as previously reported (32), and cultured in L-15 medium (Hyclone) supplemented with 20\% FBS (Fetal Bovine Serum, Gibco, Lot 1861242 ) in $4 \% \mathrm{CO}_{2}$ at $26^{\circ} \mathrm{C}$. Miiuy croaker kidney cell lines (MKC) were cultured in L-15 medium supplemented with $15 \% \mathrm{FBS}$ in $4 \% \mathrm{CO}_{2}$ at $26^{\circ} \mathrm{C}$. The Miiuy croaker macrophages and MKC cells of miiuy croaker were transfected with siRNAs or plasmids using Lipofectamin $3000^{\mathrm{TM}}$ (Invitrogen). HEK293 cells were cultured in DMEM medium which contained 
TABLE 1 | PCR primer sequence information in this study.

\begin{tabular}{|c|c|}
\hline Primers & Sequences $\left(5^{\prime}-3^{\prime}\right)$ \\
\hline \multicolumn{2}{|l|}{ Vector construction } \\
\hline TRIF-Kpnl-F & CGGGGTACC ATGAGCCGCGAGGGAGAA \\
\hline TRIF-EcoRI-R & CCGGAATTCCTAAAGACATTGCTCATC \\
\hline TRIF-GFP-Kpnl-F & CGGGGTACCATGAGCCGCGAGGGAGAAG \\
\hline TRIF-GFP-BamHI-R & CGCGGATCCCTAAAGACATTGCTCATCTG \\
\hline IRF3-Kpnl-F & CGGGGTACCATGTCTCATTCTAAACCTCTGCTCATC \\
\hline IRF3-Xbal-R & TGCTCTAGAGTGTCAGTACAGCTCCATCATCTC \\
\hline IRF3-shRNA1-F & $\begin{array}{l}\text { GATCCGCTTCAAACTGGTCTCTGATTCAAGA } \\
\text { GATCAGAGACCAGTTGAAGCTIITIG }\end{array}$ \\
\hline IRF3-shRNA1-R & $\begin{array}{l}\text { AATTCAAAAAAGCTTCAAACTGGTCTCTG } \\
\text { ATCTCTTGAATCAGAGACCAGTTTGAAGCG }\end{array}$ \\
\hline IRF3-shRNA2-F & $\begin{array}{l}\text { GATCCGACTGAAGAGCTGACTCAATTCAAG } \\
\text { AGATTGAGTCAGCTCTTCAGTCTाITG }\end{array}$ \\
\hline IRF3-shRNA2-R & $\begin{array}{l}\text { AATTCAAAAAAGACTGAAGAGCTGACTCAAT } \\
\text { CTCTTGAATTGAGTCAGCTCTTCAGTCG }\end{array}$ \\
\hline IRF3-shRNA3-F & $\begin{array}{l}\text { GATCCGGATAACATACCTGCCTTCTTCAAGA } \\
\text { GAGAAGGCAGGTATGTTATCCTाITTG }\end{array}$ \\
\hline IRF3-shRNA3-R & $\begin{array}{l}\text { АATTCAAAAAAGGATAACATACCTGCCTTCT } \\
\text { СTCTTGAAGAAGGCAGGTATGTTATCCG }\end{array}$ \\
\hline IRF3-dDBD-EcoRI-F & CCGGAATTCAACTCTAGTGCTGGATCC \\
\hline IRF3-dDBD-EcoRI-R & CCGGAATTCAGCGTAATCTGGAACATCGT \\
\hline IRF3-dIAD-EcoRI-F & CCGGAATTCCCAGACAACAGGCCTTGGGAG \\
\hline IRF3-dIAD-EcoRI-R & CCGGAATTCATCTCCATCTCTGGTCTTGTT \\
\hline IRF3-dSRD-EcoRI-F & CCGGAATTCCTCGAAGAGATGATGGAGCTG \\
\hline IRF3-dSRD-EcoRI-R & CCGGAATTCGGCGCCGCCTCCAACAGCCA \\
\hline TAK1-BamHI-F & $\begin{array}{l}\text { CTTGGTACCGAGCTCGGATCCATGTCTC } \\
\text { TAACGTTACCGTCCGC }\end{array}$ \\
\hline TAK1-EcoRI-R & $\begin{array}{l}\text { TGATGGATATCTGCAGAATTCGACATGCA } \\
\text { GGACACAGTAGAATGC }\end{array}$ \\
\hline TBK1-HindIII-F & CCCAAGCTTATGCAGAGCACCACCAACT \\
\hline TBK1-EcoRI-R & CCGGAATTCCCGTTGTTCACGAACTCA \\
\hline P65-Hindlll-F & CCCAAGCTTATGGCGGATGTGTACGGAT \\
\hline P65-Kpnl-R & CGGGGTACCCTGAAGGCTAAAGGAGCAG \\
\hline STAT1a-BamHI-F & CGCGGATCCGCGCAGTGGTGCCAGCTC \\
\hline STAT1a-EcoRI-R & CCGGAATTCTCAGTITGGTCTGGAAACTC \\
\hline \multicolumn{2}{|l|}{ Real-time PCR } \\
\hline IRF2-RT-F & CGAGGAGGTGAAGGATAAA \\
\hline IRF2-RT-R & GGATGCCTGAGATGCTGT \\
\hline IRF3-RT-F & GAATGATGCTGCTAACCC \\
\hline IRF3-RT-R & CGACTGGAGTCTCAAACG \\
\hline IRF6-RT-F & AGAAATTGGCGAGGAAGA \\
\hline IRF6-RT-R & ACAGGGCGTCAGGTAGAG \\
\hline RIP1-RT-F & GTCAAGTTGCTGGGTGTAA \\
\hline RIP1-RT-R & TCTATGATGATTCTGCCTाT \\
\hline TRAF3-RT-F & GAGGTGCCGTGTCCGTTGGGTAA \\
\hline TRAF3-RT-R & TCGCCATCATTCTCAGGTGTTCAGC \\
\hline TRAF6-RT-F & ATGATGGAAAAGGAACGGGAAT \\
\hline TRAF6-RT-R & TCGGACAGCGAACAGTTAGTGA \\
\hline TNF $\alpha-R T-F$ & GTTGCTTGGTACTGGAATGG \\
\hline TNF $\alpha-R T-R$ & TGTGGGATGATGATCTGGTTG \\
\hline IL8-RT-F & AGCAGCAGAGTCTTCGT \\
\hline IL8-RT-R & TCTTCGCAGTGGGAGTT \\
\hline IL1 $\beta-R T-F$ & CATAAGGATGGGGACAACGAG \\
\hline IL1 $\beta-R T-R$ & TAGGGGACGGACACAAGGGTA \\
\hline$\beta$-actin-RT-F & GAGCCGCACGCTTCTTा \\
\hline$\beta$-actin-RT-R & CTGCTGTAGCCGAGGAC \\
\hline
\end{tabular}

the $10 \%$ FBS, $2 \mathrm{mM}$ L-glutamine, $100 \mathrm{U} / \mathrm{ml}$ penicillin, and $100 \mathrm{mg} / \mathrm{ml}$ streptomycin, and under humidified conditions with $5 \% \mathrm{CO}_{2}$ at $37^{\circ} \mathrm{C}$. Fish EPC cells were cultured in medium 199 which contained the $10 \%$ FBS, $2 \mathrm{mM} \mathrm{L}$-glutamine, $100 \mathrm{U} / \mathrm{ml}$ penicillin, and $100 \mathrm{mg} / \mathrm{ml}$ streptomycin, and under humidified conditions with $5 \% \mathrm{CO}_{2}$ at $28^{\circ} \mathrm{C}$. HEK293 and EPC cells were transfected with various plasmids by using Lipofectamin $2000^{\mathrm{TM}}$ (Invitrogen) and X-tremeGENE HP (Roche), respectively. In addition, the proteasome inhibitor (MG132) or cycloheximide $(\mathrm{CHX})$ was added into medium at $36 \mathrm{~h}$ post-transfection.

\section{RNA Isolation and qRT-PCR Analysis}

Total RNA was isolated from miiuy croaker macrophage cells with TRIzol reagent (Takara) according to the manufacturer's instructions. The cDNA was synthesized by using the FastQuant RT Kit (Tiangen) including DNase treatment of RNA to eliminate genomic contamination. qRT-PCR was performed with a SYBR Premix Ex Taq kit (TaKaRa) on the 7500 system (Applied Biosystems, USA). PCR cycling conditions were as follows: $10 \mathrm{~s}$ at $95^{\circ} \mathrm{C}$, and followed by 40 cycles consisting of $5 \mathrm{~s}$ at $95^{\circ} \mathrm{C}$, then $31 \mathrm{~s}$ at $60^{\circ} \mathrm{C}$. All primers used for qRT-PCRs are shown in Table $\mathbf{1}$ and $\beta$-actin was used as an internal control.

\section{Luciferase Reporter Assays}

HEK293 cells were transfected with expression plasmids and NF- $\kappa B$, IFN $\beta$ or ISRE reporter gene plasmids, and Renilla luciferase reporter plasmid (pRL-TK) was used as the internal control. The proportion of the amount of plasmids: pRL-TK: NF- $\kappa B$, IFN $\beta$, IFNr, or ISRE reporter gene plasmids are 1:10. Reporter luciferase activities were measured by using the dual luciferase reporter assay system (Promega). The control group used the equal amount of corresponding empty vector compared with the experimental group. For each experiment, the results were done in triplicate for each experiment, and three independent experiments were conducted.

\section{Prokaryotic Expression and Polyclonal Antiserum}

The full-length CDS region of miiuy croaker TRIF was cloned into EcoR I/Xho I sites of pGEX-4T-1 vector (GE) to construct pGEX-4T-1-TRIF plasmid. Then, the plasmid pGEX-4T-1-TRIF was transformed into the BL21 (DE3) Escherichia coli strain and expressed as a protein containing TRIF fused with GST. The fusion protein was induced by isopropyl $\beta$-D-thiogalactoside and purified by GST-Bind resin chromatography. The purified fusion protein was applied to immunize New Zealand White rabbits to raise a polyclonal anti-TRIF antiserum (31).

\section{Immunoblot Assays}

The HEK293 cells were washed by using cold PBS and were lysed by using western and IP cell lysis buffer. Protein concentrations of the cell lysates were measured by BCA assay (Pierce) method and equalized with the extraction reagent. Equal amount of the extracts mixed with equal amount of $2 \times$ SDS loading buffer and loaded to SDS-PAGE, which subsequently transferred onto PVDF 
membranes (Millipore) using semi-dry (Bio-Rad Trans Blot Turbo System). Then the membranes were blocked for $90 \mathrm{~min}$ at room temperature in $5 \%$ dried skimmed milk and were incubated primary antibodies at $4^{\circ} \mathrm{C}$ overnight. The primary antibodies used in this study were against HA, Flag, Myc, GFP Tag (Santa Cruz, mouse), and GAPDH, $\beta$-actin, tubulin (Sigma, mouse), and TRIF (Sigma, rabbit). Then, the membranes were three times with TBST and then incubated with the secondary antibody conjugated with horseradish peroxidase (Beyotime, mouse/rabbit) for $60 \mathrm{~min}$ at room temperature. The immunoreactive proteins were detected with WesternBright ${ }^{\mathrm{TM}}$ ECL (Advansta), and digital imaging was performed with a cold CCD camera.

\section{Immunoprecipitation Assay}

For immunoprecipitation (IP) experiments, HEK293 cells were seeded onto $10 \mathrm{~cm}^{2}$ plate overnight then were co-transfected with $5 \mu \mathrm{g}$ indicated plasmids. At $48 \mathrm{~h}$ post-transfection, the cells were washed three times with ice-cold PBS. Then the cells were lysed with $500 \mu \mathrm{l}$ western and IP lysis buffer (Beyotime) containing protease inhibitor cocktail (Bitake) at $4^{\circ} \mathrm{C}$ for $30 \mathrm{~min}$ on a rocker platform. Then the cellular debris was removed by centrifugation at $14,000 \mathrm{~g}$ for $15 \mathrm{~min}$ at $4^{\circ} \mathrm{C}$. After centrifugation, the supernatant was transferred into a fresh centrifuge tube and incubated with $50 \mu \mathrm{l}$ protein $\mathrm{A}+\mathrm{G}$ (Sigma) together with $1 \mu \mathrm{g}$ monoclonal antiFlag (Sigma) overnight at $4^{\circ} \mathrm{C}$ with constant and softly agitation. The following day, the IP protein was collected by centrifugation at $2,500 \mathrm{~g}$ for $5 \mathrm{~min}$ at $4^{\circ} \mathrm{C}$. Then beads were washed five times with western and IP lysis buffer and resuspended in $60 \mu \mathrm{l} 2 \times$ SDS loading buffer. The immunoprecipitates and whole-cell lysates (WCLs) were analyzed by immunobloting.

\section{Fluorescent Microscopy}

HEK293 cells were seeded onto 24-well plate and transfected by using Lipofectamine ${ }^{\mathrm{TM}} 2000$ (Invitrogen) with indicated plasmids for $48 \mathrm{~h}$. Then the images were obtained under a fluorescence microscope (Leica).

\section{RNA Interference}

The miiuy croaker IRF3-specific siRNA (si-IRF3) and TRIF-specific siRNA (si-TRIF) were 5'-GCUUCAAACUGGUCUCUGATT-3' (sense), 5'-UCAGAGACCAGUUUGAAGCTT-3' (antisense), and 5'-GAGACAACUACCUUGCUAGTT-3' (sense), 5' -CUAG CAAGGUAGUUGUCUCTT-3' (antisense), respectively. The scrambled control RNA (si-Ctrl) sequences were 5'-UUCUCC GAACGUGUCACGUTT-3' (sense) and 5'-ACGUGACACGU UCGGAGAATT-3' (antisense). Miiuy croaker macrophages or HEK293 cells were transfected with $50 \mathrm{nM}$ of each siRNA for up to $36 \mathrm{~h}$ before SCRV stimulation using Lipofectamine $3000^{\mathrm{TM}}$.

\section{Statistical Analysis}

All the experiments were performed at least three independent experiments $(n \geq 3)$ with three technical replicates for each
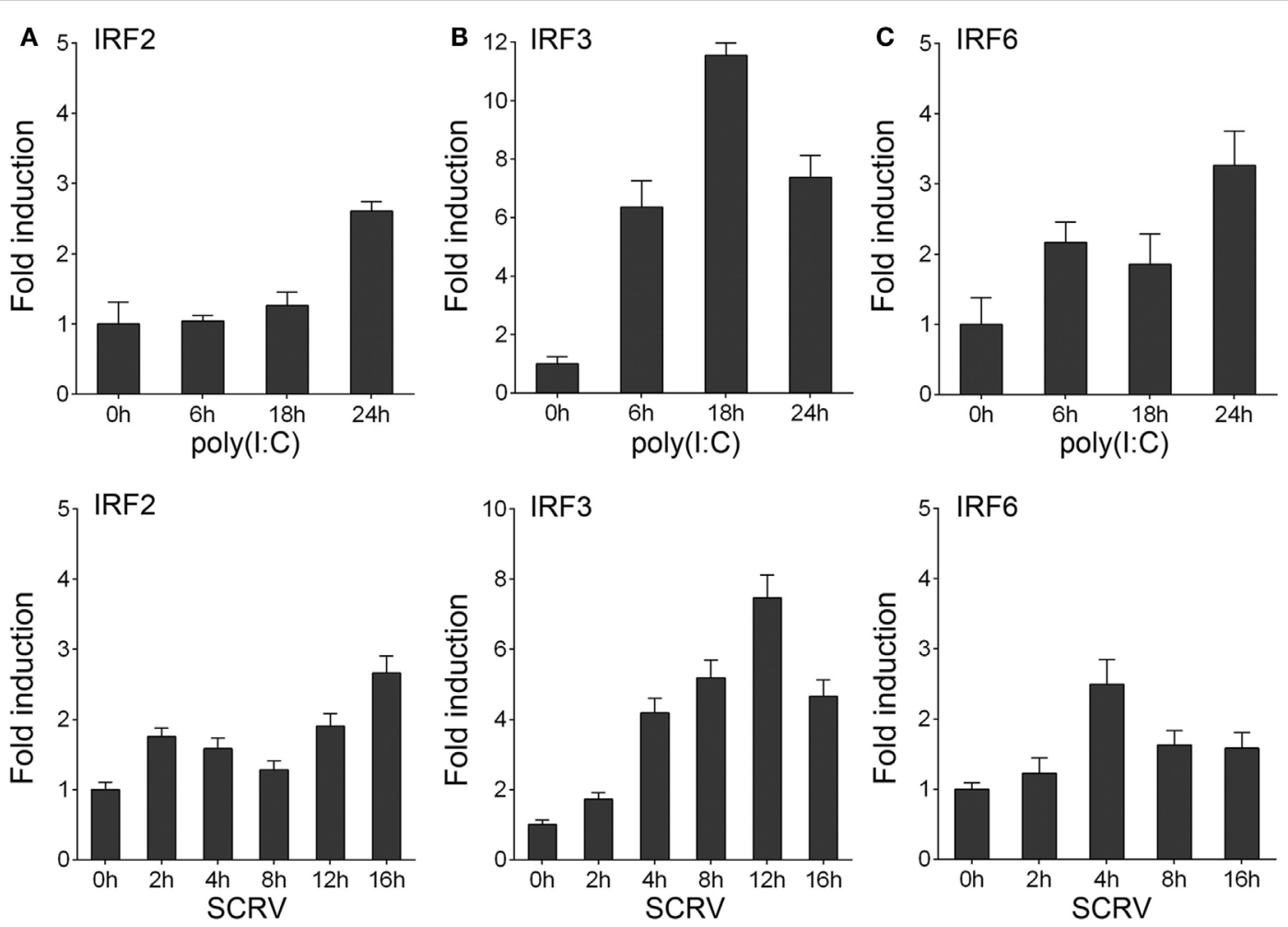

FIGURE 1 | Inducible expression patterns of IRF3. (A-C) Miiuy croaker macrophages were seeded onto 12-well plate overnight and stimulated with poly(l:C) $(2 \mu \mathrm{g} / \mathrm{ml})$ for 6,18 , and $24 \mathrm{~h}$, or SCRV $(\mathrm{MOI}=5)$ for $2,4,8,12$, and $16 \mathrm{~h}$. Then total RNAs were extracted to examine the expression of IRF2, IRF3, and IRF6 by qRT-PCR. The data were normalized to $\beta$-actin. Results are standardized to 1 in control cells. 
experiment. The relative gene expression data was obtained by using the $2^{-\Delta \Delta \mathrm{CT}}$ method, and comparisons between groups were analyzed by one-way analysis of variance followed by Duncan's multiple comparison tests (33). Results are expressed as mean \pm SE, and differences between means were with $p$ values of $<0.05$ considered to be statistically significant.

\section{RESULTS}

\section{IRF3 Is Upregulated After Poly(I:C) and SCRV Induction}

To determine whether the expression of host IRF3 regulated by viral infection, miiuy croaker macrophages were stimulated with polyriboinosinic polyribocytidylic acid [poly(I:C)] or infected with fish rhabdovirus SCRV. SCRV is a double-stranded RNA virus, which is used as a presumably physiological form of poly(I:C). The expression of IRF3 was then detected by qRT-PCR. The results showed that IRF3 expression was upregulated in both conditions. The results also suggested that IRF3 could be activated to a greater extent after poly(I:C) stimulation and SCRV infection in host cell than after the administration of IRF2 and IRF6 (Figure 1). These data demonstrated the important role of IRF3 in viral infection.

\section{IRF3 Inhibits the NF-кB Signaling Pathway}

To identity the regulatory role of IRF3, IRF3 was transfected into HEK293 cells together with different reporter gene (NF- $\kappa B$, IFN $\beta$,
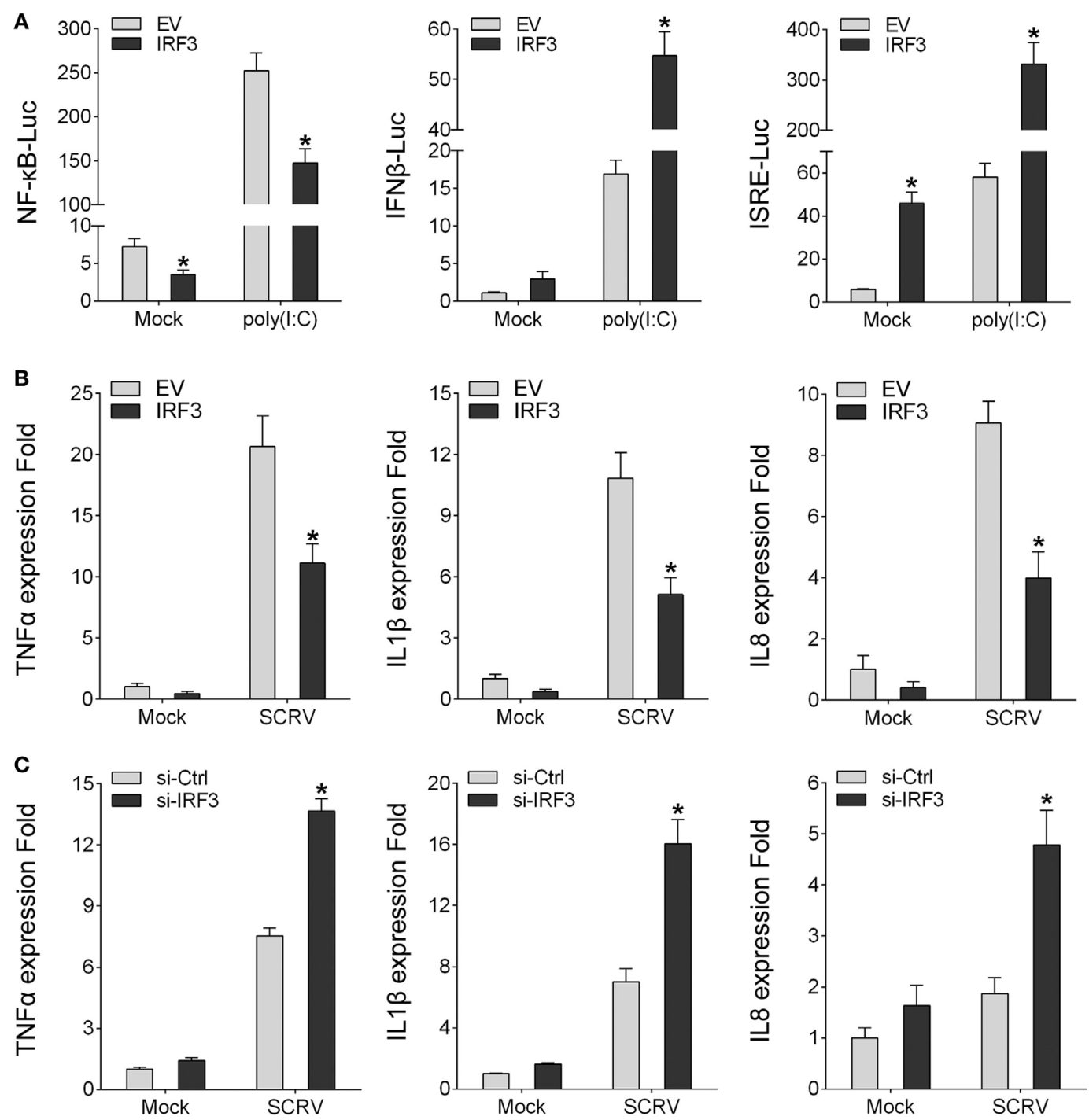

FIGURE 2 | IRF3 can inhibit poly(I:C)-induced NF-кB pathway and expression of downstream cytokines. (A) HEK293 cells were co-transfected with empty vector or IRF3 together with NF-kB, IFN $\beta$, or ISRE reporter gene, after $36 \mathrm{~h}$, stimulated with poly(l:C) for $12 \mathrm{~h}$, then the luciferase activity was measured, the luciferase activity value was achieved against the Renilla luciferase activity. (B) Miiuy croaker macrophages were transfected with empty vector or IRF3, after $36 \mathrm{~h}$, infected with SCRV $(\mathrm{MOI}=5)$ for $12 \mathrm{~h}$, then total RNAs were extracted to examine the expression of TNF $\alpha$, IL1 $\beta$, and IL 8 by qRT-PCR. (C) Miiuy croaker macrophages were seeded on 24-well plate overnight and transfected with the scrambled control RNA or si-IRF3, after $36 \mathrm{~h}$, infected with SCRV for $12 \mathrm{~h}$, then total RNAs were extracted to examine the expression of TNF $\alpha, I L 1 \beta$, and IL8 by qRT-PCR. ${ }^{*} p<0.05$ versus the controls. 
and ISRE), respectively, then the HEK293 cells were treated with poly(I:C). The result showed that, among the signaling pathways induced by poly(I:C), only NF- $\kappa$ B signaling pathway was inhibited by IRF3 (Figure 2A). To further confirm the role of IRF3, IRF3 plasmids were transfected into miiuy croaker MKC cells and then infected with SCRV. qRT-PCR analysis indicated that IRF3 overexpression inhibited the transcription of downstream cytokines of NF- $\kappa \mathrm{B}$ signaling pathway, including TNF $\alpha$, IL1 $\beta$, and IL8 (Figure 2B). Furthermore, IRF3-siRNA was transfected into the macrophage and qRT-PCR was performed to assess the expression of TNF $\alpha$, IL1 $\beta$, and IL8 cytokines in the IRF3 knockdown cells. SCRV treatment increased TNF $\alpha$, IL1 $\beta$, and IL8 expression at $12 \mathrm{~h}$ post-infection in the IRF3 knockdown cells as compared with the control cells (Figure 2C). These data suggested that IRF3 might inhibit poly(I:C)-induced NF- $\mathrm{KB}$ signaling pathway.

\section{IRF3 Inhibits the TRIF-Mediated NF-kB Signaling Pathway}

To identity the role of IRF3 in NF- $\kappa B$ signaling pathway, first, we determined whether IRF3 can affect the TRIF-mediated NF- $\kappa B$ signaling pathway. According to the results of luciferase reporter assays, TRIF expression sufficiently activated the NF- $\kappa \mathrm{B}$ reporter, and the NF- $\kappa \mathrm{B}$ activation was suppressed by co-transfected with IRF3 plasmid compared to control. However, co-transfection with TRIF and IRF3 plasmids was unable to inhibit IFN $\beta$, IFNr, and ISRE reporter genes (Figure 3A). To further determine whether IRF3 can negatively regulate TRIF-mediated NF- $\kappa B$ signaling pathway, we performed the IRF3 concentration gradient and different time points experiments with overexpression of TRIF by NF- $\kappa$ B reporter assays (Figure $3 B$ ). Overall, these results indicated that a negative regulation effect was intensified at increased amount of IRF3 expression plasmid. In addition, the results showed that IRF3 could not inhibit NF- $\kappa \mathrm{B}$ pathway mediated by TRIF downstream genes (Figure 3C). Consequently, IRF3 can inhibit TRIF-mediated NF- $\kappa$ B signaling pathway.

\section{IRF3 Promotes TRIF Degradation}

To investigate the effect of IRF3 on TRIF expression, HEK293 cells were co-transfected with TRIF and IRF3 or negative control STAT1a plasmids, after $48 \mathrm{~h}$ transfection, the TRIF expression
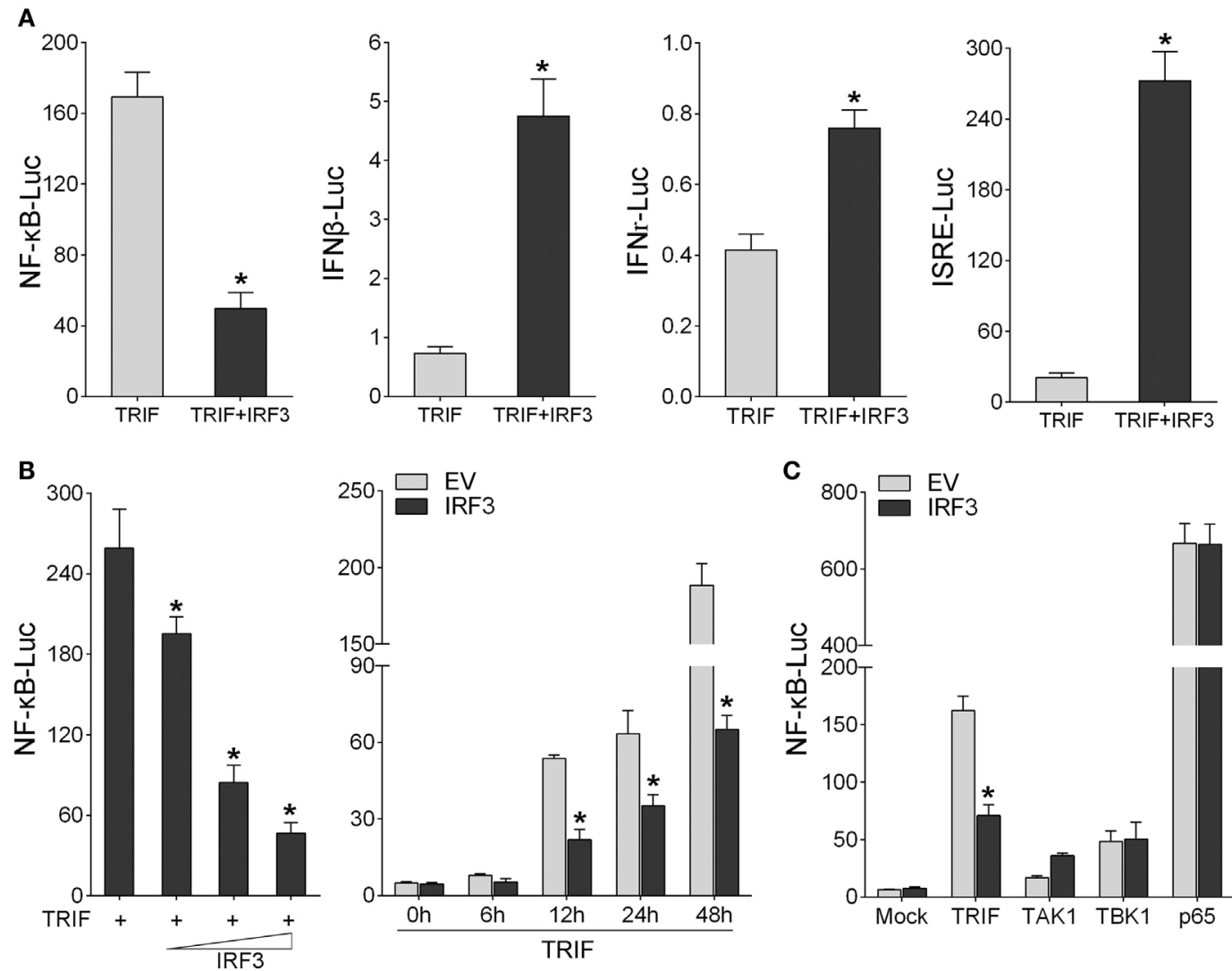

FIGURE 3 | IRF3 represses TRIF-mediated NF-кB signaling pathway. (A) HEK293 cells were co-transfected with TRIF and empty vector or IRF3 together with $\mathrm{NF}-\kappa \mathrm{B}$, IFN $\beta$, IFNr, or IFN stimulatory response elements reporter gene (ISRE), after $48 \mathrm{~h}$, the luciferase activity was measured. (B) The concentration gradient experiment of IRF3 expression plasmid within TRIF and NF- $\mathrm{kB}$ reporter gene was conducted (left). After $48 \mathrm{~h}$, the luciferase activity was measured. After co-transfected with TRIF and IRF3 expression plasmids, together with NF-kB reporter gene, the luciferase activity was measured at different time points (right). (C) HEK293 cells were co-transfected with NF-кB reporter gene and empty vector or IRF3, together with TRIF, TAK1, TBK1, or p65, after 48 h, the luciferase activity was measured. The luciferase activity value was achieved against the Renilla luciferase activity. ${ }^{\star} p<0.05$ versus the controls. 
was examined by immunoblot assays. The results indicated that the protein levels of TRIF were decreased in the presence of IRF3; however, STAT1a had no effect on TRIF protein levels (Figure 4A). To confirm the effect of IRF3 on TRIF expression in fish cells, the EPC cells were co-transfected with TRIF and IRF3 plasmids, after $48 \mathrm{~h}$ transfection, the TRIF expression was examined by immunoblot assays. The results indicated that the protein levels of TRIF also decreased in the presence of IRF3 in fish cells (Figure 4B). To further confirm the effect of endogenous IRF3 on TRIF expression in miiuy croaker, the miiuy croaker macrophages were transfected with IRF3-siRNA which was used by inhibiting IRF3 expression. Then the expression of endogenous TRIF protein was examined by immunoblot assays. The results showed that TRIF protein could be upregulated when IRF3-siRNA was transfected (Figure 4C). To further confirmed the role of IRF3 on TRIF expression, HEK293 cells were cotransfected with TRIF-GFP expression plasmid together with IRF3 or the empty vector. As shown in Figure 4D, the fluorescence signals of TRIF-GFP markedly decreased in the presence of IRF3 compared with empty vector. Then the cells were lysed and TRIF protein was examined by immunoblot assays with GFP antibody. Based on the assay results, we speculated that IRF3 may promote TRIF degradation. Accordingly, we conducted a concentration gradient in HEK293 or EPC cells respectively; (Figure 4E) and time point experiments (Figure 4F), and the results demonstrated that IRF3 specifically targeted TRIF for protein degradation.

\section{IRF3 Affects TRIF Downstream Molecules}

To detect the influence on IRF3 for TRIF downstream molecules, the miiuy croaker MKC cells or macrophages were transfected IRF3 plasmid or IRF3-siRNA respectively, cells were infected SCRV after $36 \mathrm{~h}$ transfection, then qRT-PCR was performed. The results showed that the expression of TRIF downstream genes (RIP1, TRAF3, and TRAF6) were downregulated after IRF3 was transfected into the MKC cells (Figure 5A). However, when the cells were transfected with IRF3-siRNA, the expression of RIP1 and TRAF3 were upregulated (Figure 5B). The results indicated that IRF3 can affect the expression of TRIF downstream molecules. The above results indicated that IRF3

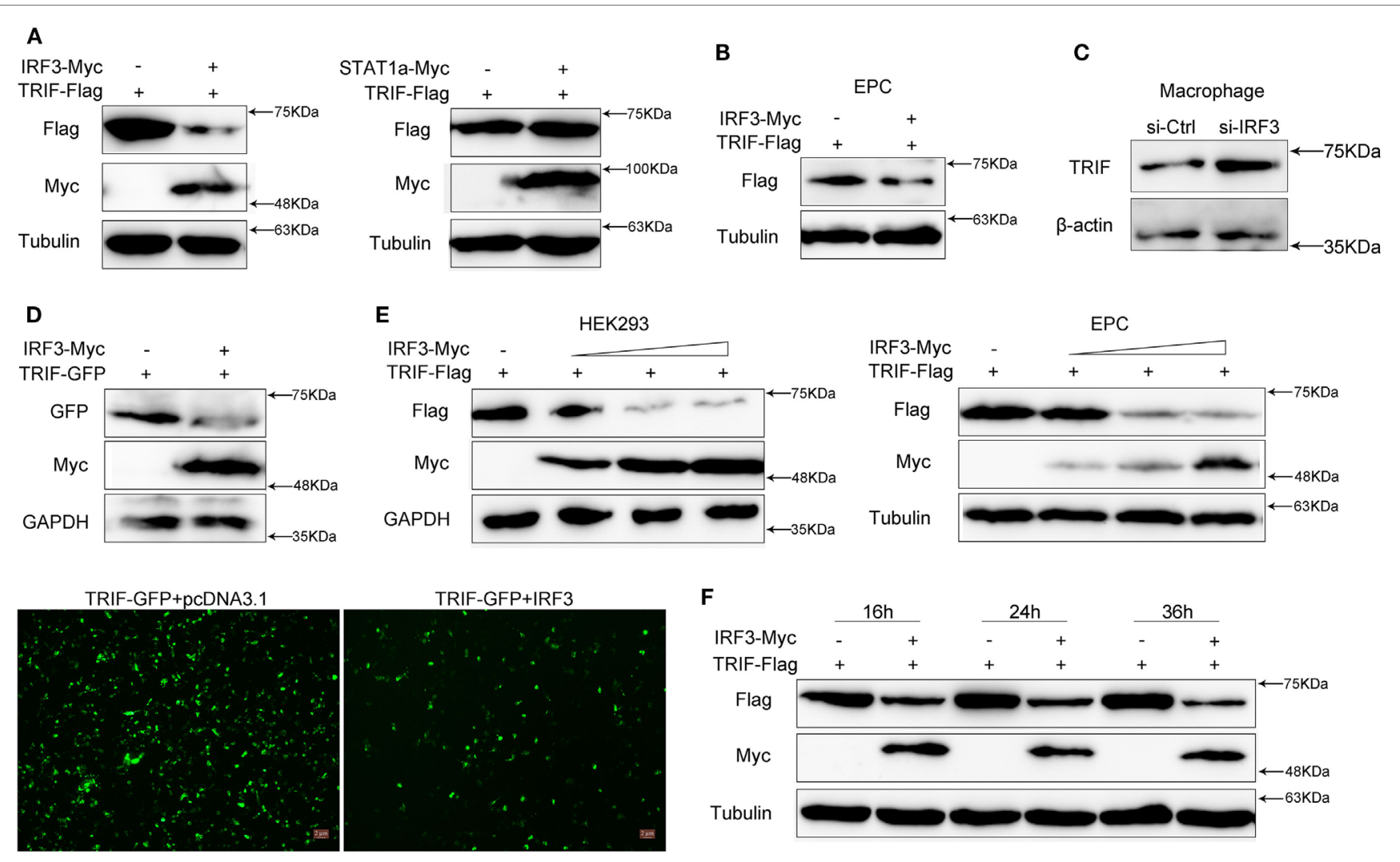

FIGURE 4 | IRF3 promotes the TRIF degradation. (A) HEK293 cells were co-transfected with empty vector, IRF3 (left) or STAT1a (right) together with TRIF, after $48 \mathrm{~h}$, TRIF protein levels were determined by immunoblot assays and normalized to tubulin. (B) EPC cells were co-transfected with empty vector or IRF3 together with TRIF, after $48 \mathrm{~h}$, TRIF protein levels were determined by immunoblot assays and normalized to tubulin. (C) Miiuy croaker macrophages were transfected with the scrambled control RNA (si-Ctrl) or si-IRF3. After $48 \mathrm{~h}$, TRIF protein levels were determined by Western blot and normalized to $\beta$-actin. (D) HEK293 cells were co-transfected with empty vector, IRF3 together with TRIF-GFP, after $48 \mathrm{~h}$, the fluorescence signals of TRIF-GFP were detected by fluorescence microscopy (below), then TRIF protein levels were determined by immunoblot assays and normalized to GAPDH (top). (E) The concentration gradient experiment of IRF3 expression plasmid within TRIF was conducted in HEK293 (left) and EPC cells (right) respectively, TRIF protein levels were determined by immunoblot assays and normalized to GAPDH or tubulin. (F) The time gradient experiment of IRF3 expression plasmid within TRIF was conducted, TRIF protein levels were determined by immunoblot assays, and normalized to tubulin. 

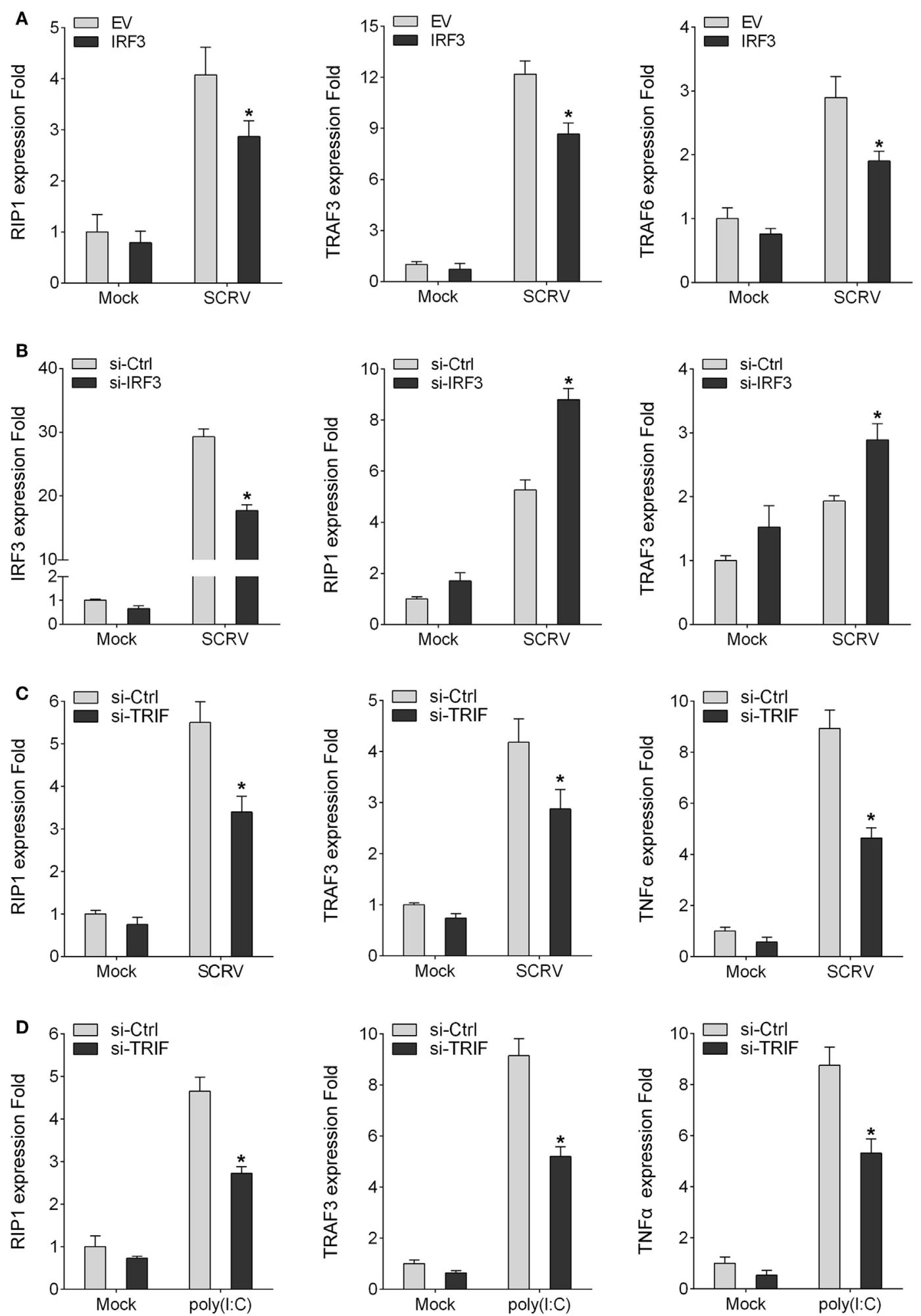

FIGURE 5 | IRF3 affects TRIF downstream molecules. (A) MKC were seeded onto 24-well plate overnight and transfected with empty vector or IRF3, after 36 h, infected with SCRV for $12 \mathrm{~h}$, then total RNAs were extracted to examine expression of RIP1, TRAF3, and TRAF6 by qRT-PCR. (B) Miiuy croaker macrophages were transfected with the scrambled control RNA (si-Ctrl) or si-IRF3, after $36 \mathrm{~h}$, infected with SCRV for $12 \mathrm{~h}$, then total RNAs were extracted to examine expression of IRF3, RIP1, and TRAF3 by qRT-PCR. (C,D) Miiuy croker macrophages were transfected with si-Ctrl or si-TRIF. After 36 h, macrophages were then infected with SCRV for $12 \mathrm{~h}$ and the expression of RIP1, TRAF3, and TNF $\alpha$ and stimulated with poly(l:C) for another $12 \mathrm{~h}$ and the expression of RIP1, TRAF3, and TNF $\alpha$ were determined. * $p<0.05$ versus the controls. 
promoted TRIF degradation and affected TRIF downstream molecules. To further determine that TRIF protein can be degraded and whether their degradation affected the expression of downstream proteins and cytokines, miiuy croaker-specific TRIF-siRNA was transfected into macrophages. At $36 \mathrm{~h}$ posttransfection, the cells were treated with poly(I:C) or infected SCRV for $12 \mathrm{~h}$, then qRT-PCR was subsequently performed. The results showed that the downstream proteins and cytokines of TRIF were downregulated when the cells were transfected with TRIF-siRNA (Figures 5C,D). These dattum indicated that knockdown of TRIF significantly decreased the expression of the downstream proteins and cytokines after poly(I:C) stimulation or SCRV infection in the macrophages.

\section{Knockdown of IRF3 Potentiates TRIF-Mediated NF- $\kappa B$ Activation}

To further confirm the function of IRF3 in the TRIF-mediated NF- $\kappa \mathrm{B}$ pathway, we investigated whether knockdown of IRF3 affected TRIF-mediated NF- $\kappa \mathrm{B}$ activation. First, we synthesized and constructed three knockdown plasmids designated as IRF3-shRNA1 (IRF3-sh1), IRF3-shRNA2 (IRF3-sh2), and IRF3shRNA3 (IRF3-sh3), respectively. Then we confirmed that three IRF3-shRNA plasmids efficiently downregulated the expression of IRF3 through immunoblot assays (Figure 6A). HEK293 cells were co-transfected with TRIF, IRF3, and IRF3-shRNA1 plasmids, at post-transfection $48 \mathrm{~h}$, the TRIF expression was then examined by immunoblot assays. The result showed that

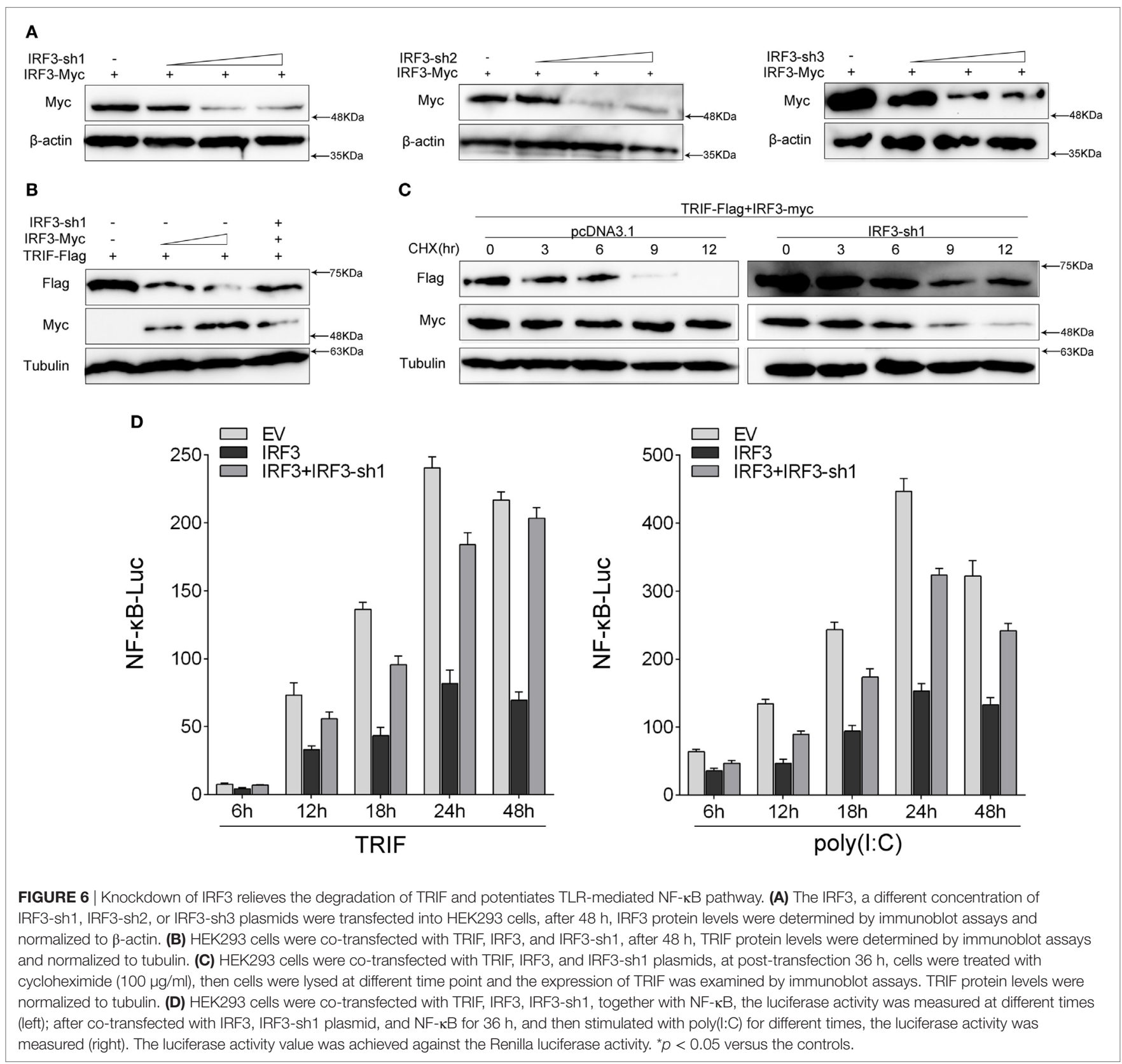


IRF3-shRNA inhibited IRF3 expression, thereby weakening TRIF degradation (Figure 6B). HEK293 cells were co-transfected with TRIF, IRF3, and IRF3-shRNA1 plasmids, at post-transfection $36 \mathrm{~h}$, and then cells were treated with CHX before cells were lysed. The TRIF protein expression was then examined by immunoblot assays. The results also showed that IRF3-shRNA inhibited IRF3 expression, thereby weakening TRIF degradation (Figure 6C). To demonstrate the inhibitory effect of IRF3 gene on TRIF-mediated NF- $\kappa \mathrm{B}$ signaling pathway, IRF3 and IRF3-shRNAs plasmids were co-transfected into HEK293 cells together with TRIF or subsequently subjected to poly(I:C) stimulation after transfection $36 \mathrm{~h}$, and a time gradient experiment was then conducted to confirm the above results, and the data showed that the inhibitory effect of IRF3 was constant at different time points (Figure 6D). Overall, our findings indicate that IRF3 plays a negative regulator role in the TRIF-mediated NF- $\kappa$ B pathway. Furthermore, the negative regulation was weakened when IRF3-shRNA plasmids were cotransfected together with IRF3 and TRIF.

\section{IRF3 Promotes TRIF Degradation Through Proteasome Pathway}

To determine which way was used to promote TRIF degradation by IRF3, we co-transfected with TRIF and IRF3 plasmids into HEK293 cells. After $36 \mathrm{~h}$, the protein degradation was prevented by treatment with MG132. The results showed that MG132 blocked TRIF degradation (Figure 7A). TRIF was degradated in a dose-dependent manner with increasing of IRF3, but TRIF protein still can be rescued after MG132 treatment (Figure 7B). Then HEK293 cells were co-transfected with TRIF and IRF3 plasmids, at $36 \mathrm{~h}$ post-transfection, the cells were treated with $\mathrm{CHX}$, meanwhile, experimental group cells were treated with MG132 (Figure 7C), the results suggested that IRF3 could promote the TRIF degradation, whereas MG132 could impede the process. Furthermore, HEK293 cells were co-transfected with TRIF and IRF3 plasmids together with NF- $\kappa B$ reporter gene, after $36 \mathrm{~h}$ transfection, the cells were treated with MG132 for $12 \mathrm{~h}$ before cells were lysed. The activation of NF- $\kappa \mathrm{B}$ was subsequently measured by luciferase assays (Figure 7D), and the results indicated that MG132 could relieve the inhibitory effect of IRF3 on TRIF-mediated NF- $\kappa$ B signaling pathway. These results indicated that IRF3 could promote TRIF degradation through proteasome pathway, thereby weakening NF- $\kappa \mathrm{B}$ activation.

\section{Inhibitory Effect of IRF3 Primary Depends on IAD Domain}

To further confirm which domain of IRF3 gene promoted TRIF degradation, which resulted in the disruption of TRIF-mediated NF- $\kappa \mathrm{B}$ activation, we constructed the mutants of IRF3, including

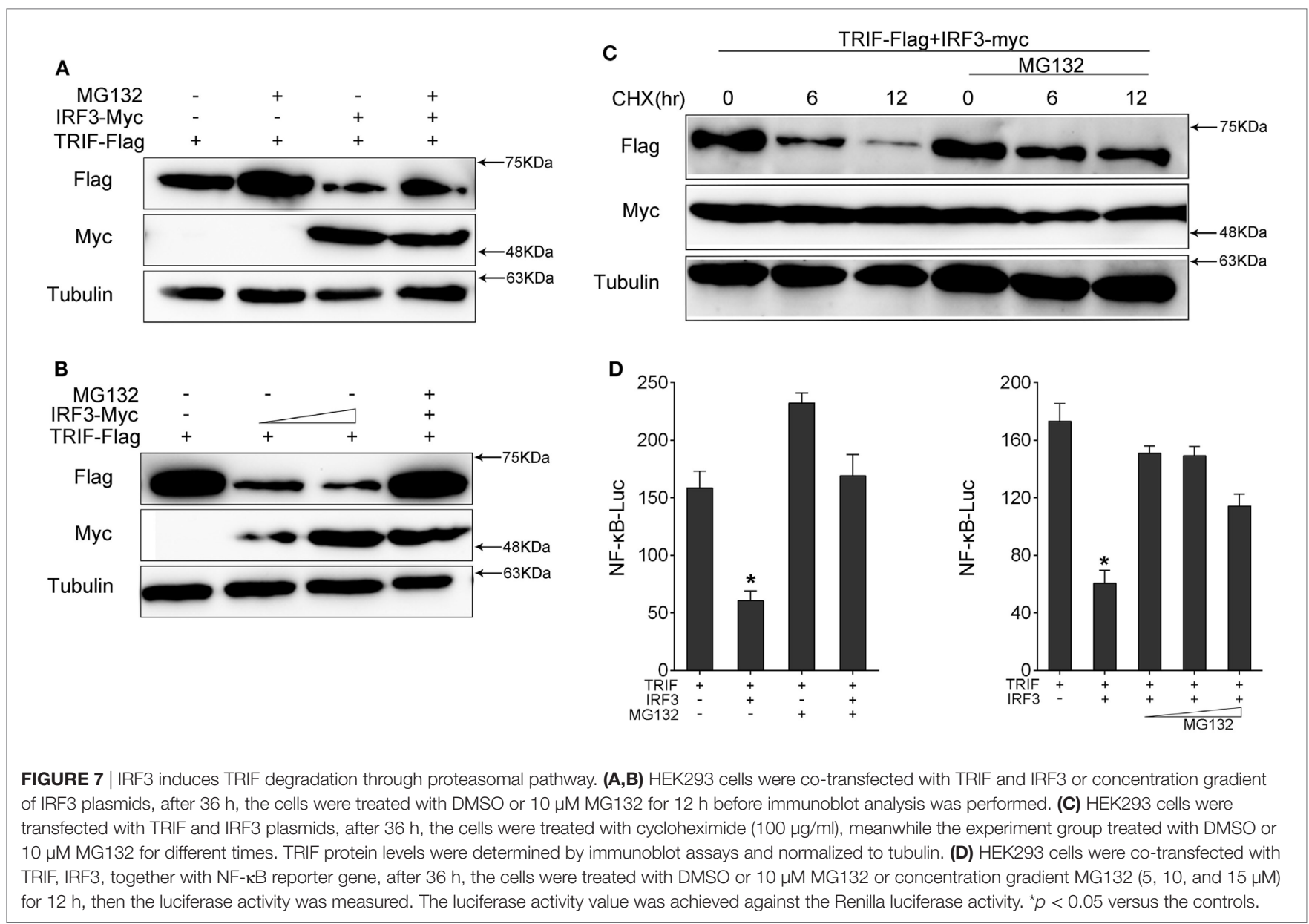


IRF3 $\Delta$ DBD $(\Delta \mathrm{DBD})$, IRF3 $\Delta \mathrm{IAD}(\Delta \mathrm{IAD})$, and IRF3 $\Delta$ SRD $(\triangle \mathrm{SRD})$ (Figure 8A). HEK293 or EPC cells were co-transfected with IRF3 or other IRF3 mutation plasmids respectively together with TRIF, after $48 \mathrm{~h}$ transfection, the expression of TRIF protein was examined by immunoblot assays. The results indicated that IRF3 promoted TRIF degradation, which depended on the IAD domain (Figure 8B). The wild type and mutants of IRF3 and TRIF along with NF- $\mathrm{KB}$ reporter gene were co-transfected into cells, the luciferase assay results also demonstrated the IAD domain of IRF3 was the main inhibition domain in the TRIFmediated NF- $\mathrm{kB}$ pathway (Figure 8C), while the deletion of the DBD and SRD domain of IRF3 showed no effect on suppressing TRIF-mediated NF-KB pathway. Additionally, the concentration gradient experiments were conducted to confirm the above results. The data suggested that the deletion IAD domain of IRF3 cannot inhibit TRIF-mediated NF- $\mathrm{kB}$ pathway. In addition, HEK293 cells were co-transfected with TRIF and IRF3 or IRF3 IAD plasmid respectively, at $36 \mathrm{~h}$ post-transfection, the cells were treated with CHX. The results suggested that IRF3 could promote the TRIF degradation, while IRF $3 \Delta \mathrm{IAD}$ cannot promote TRIF degradation (Figure 8D). Furthermore, HEK293 cells were co-transfected with TRIF and IRF3 or mutants of IRF3 plasmids together with NF- $\mathrm{kB}$ reporter gene were treated with cells for $12 \mathrm{~h}$ with MG132 after $36 \mathrm{~h}$ transfection. Then cells were lysed and the activation of NF-kB was measured by luciferase assays. The results also showed that inhibitory effect of IRF3 primary depended on IAD domain (Figure 8E). The above results indicated that IAD domain is primary domain of IRF3 that promoted TRIF degradation and the IAD domain of IRF3 inhibited the TRIF-mediated NF- $\mathrm{kB}$ signaling pathway through promoting TRIF degradation.

\section{IRF3 Leads to Elevation of TRIF Polyubiquitination and Shortens Its Half-Life}

Given that MG132 treatment can restore TRIF protein abundance in cells with IRF3 plasmid, we speculated that the TRIF ubiquitination levels in the cells would increase. To determine whether IRF3 promoted TRIF polyubiquitination, HEK293 cells were co-transfected with ubiquitination plasmid together with TRIF and IRF3 plasmids before the cells were lysed for IP with
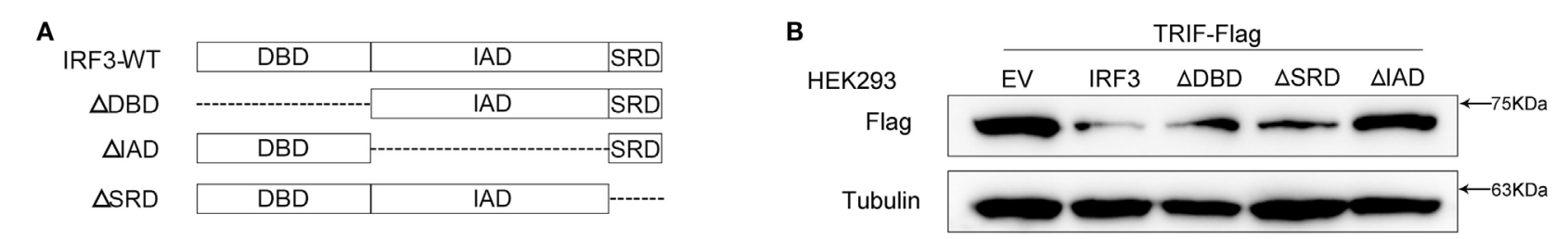
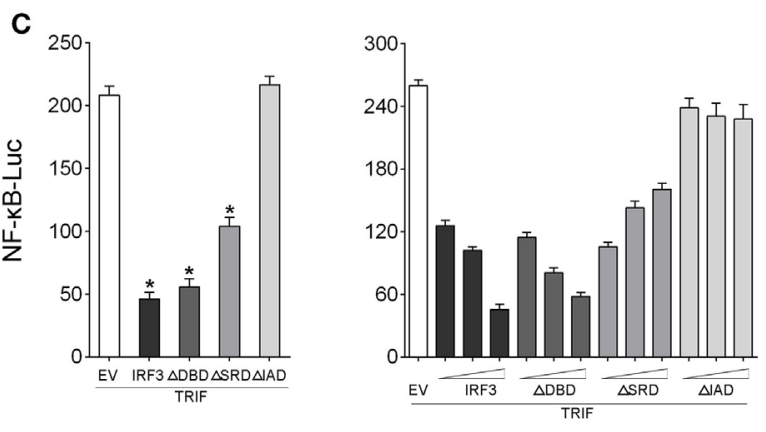

D

TRIF-Flag

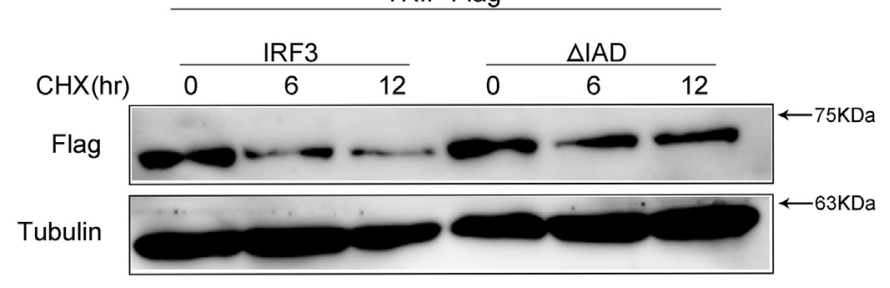

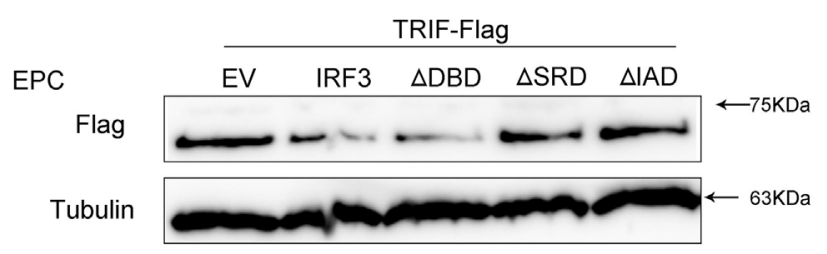

E

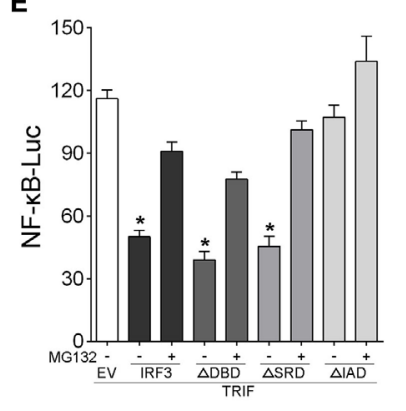

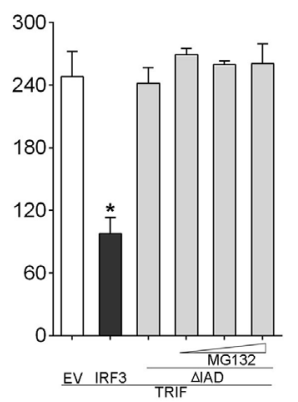

FIGURE 8 | IRF association domain (IAD) of IRF3 is critical for TRIF degradation. (A) Schematic diagram of WT and mutants of IRF3 plasmids. (B) HEK293 and EPC cells were co-transfected with IRF3, $\triangle \mathrm{DNA}$ binding domain (DBD), $\Delta \mathrm{IAD}$, or $\triangle \mathrm{SRD}$, respectively, together with TRIF, after $48 \mathrm{~h}$, TRIF protein levels was determined by immunoblot assays and normalized to tubulin. (C) HEK293 cells were co-transfected with TRIF, IRF3, $\triangle \mathrm{DBD}, \Delta \mathrm{SRD}, \Delta \mathrm{IAD}$, or a different concentration IRF3 and the above mutant's plasmid, together with NF-кB reporter gene. At $48 \mathrm{~h}$ post-transfection, the luciferase activity was measured. (D) HEK293 cells were co-transfected with TRIF and IRF3 or $\Delta$ IAD plasmids, after $36 \mathrm{~h}$, the cells were treated with cycloheximide (100 $\mu \mathrm{g} / \mathrm{ml})$ for different times, then TRIF protein levels were determined by immunoblot assays and normalized to tubulin. (E) HEK293 cells were co-transfected with TRIF, IRF3, $\Delta \mathrm{DBD}, \Delta \mathrm{IAD}$, or $\Delta \mathrm{SRD}$ respectively, together with $\mathrm{NF}-\mathrm{kB}$, after $36 \mathrm{~h}$, the cells were treated with DMSO or $10 \mu \mathrm{M} \mathrm{MG} 132$ or concentration gradient MG132 (5, 10 , and $15 \mu \mathrm{M})$ for $12 \mathrm{~h}$, then the luciferase activity was measured. The luciferase activity value was achieved against the Renilla luciferase activity. ${ }^{\star} p<0.05$ versus the controls. 

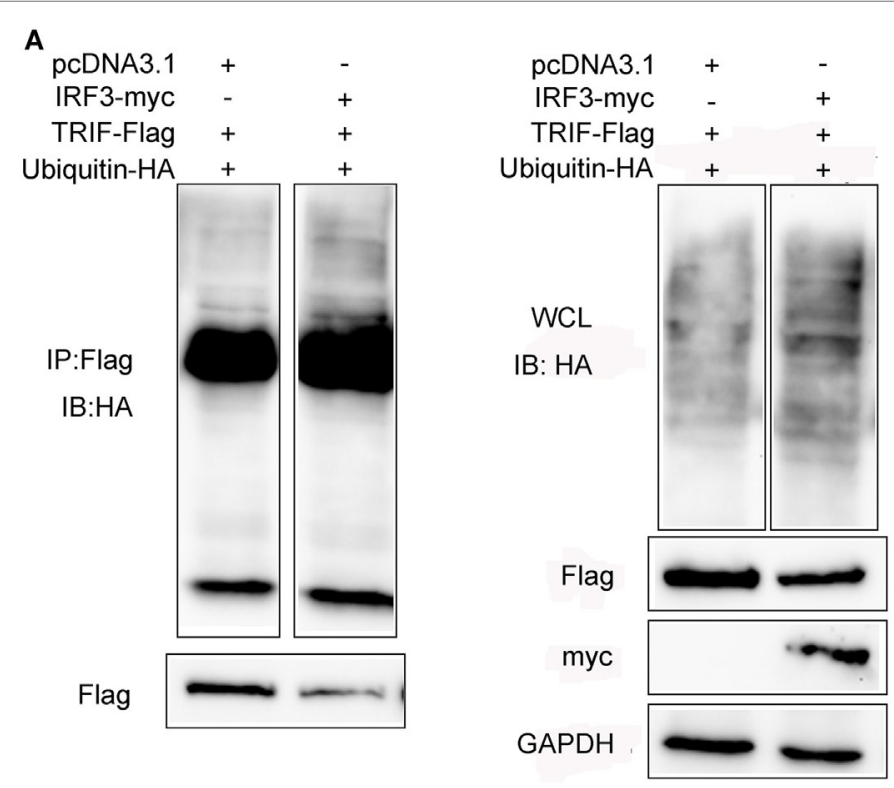

B
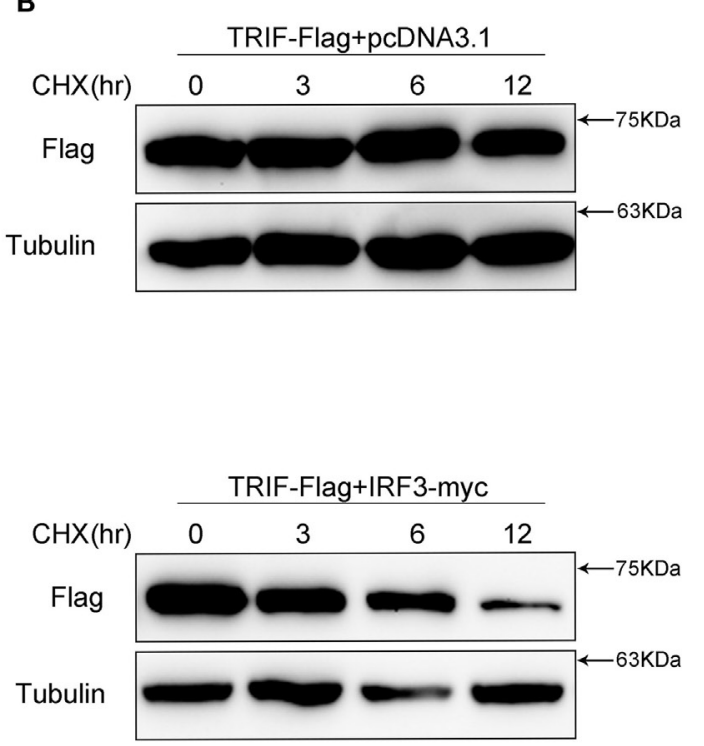

FIGURE 9 | IRF3 leads to TRIF degradation by the ubiquitin-proteasomal pathway. (A) HEK293 cells were co-transfected with TRIF, IRF3, and ubiquitin-HA plasmids, after $48 \mathrm{~h}$ post-transfection, the cells were lysed and immunoprecipitation analysed with Flag antibody and then WB with antibody against HA. Samples of whole-cell lysate were included as controls. The relative levels of ubiquitinated TRIF are shown below the images after normalization with TRIF level. (B) HEK293 cells were transiently co-transfected with TRIF and IRF3 plasmids, after $36 \mathrm{~h}$, the cells were treated with cycloheximide (100 $\mu \mathrm{g} / \mathrm{ml})$ and harvested at different times, followed by immunoblot assays with antibodies against Flag and tubulin. The relative levels of TRIF are shown below the images after normalization with tubulin.

an antibody against Flag-TRIF. The results of immunoblot assays with HA antibody showed that ubiquitinated TRIF in the cells with IRF3 increased to a greater extent than those in the cells transfected with the empty vector (Figure 9A) and indicated the intensified polyubiquitination of TRIF in the cells with IRF3 expression. Furthermore, the WCL was also detected in the blotting (Figure 9A). The above results indicated that IRF3 induced the elevation of TRIF polyubiquitination in the cells. To test whether the half-life would be shortened, HEK293 cells were co-transfected with TRIF and IRF3 plasmids. The cells were then treated with $\mathrm{CHX}$ before they were lysed at different time points. Immunoblotting was subsequently performed. In the presence of IRF3, the TRIF level decreased at a higher rate than those in the cells transfected with the empty vector (Figure 9B). At $3,6,9$, and $12 \mathrm{~h}$ after the CHX treatment, the protein levels of TRIF in the cells with IRF3 expression were degraded rapidly. By contrast, TRIF in the cells transfected with the empty vector were degraded relatively slow (Figure 9B). This result also suggested that IRF3 expression can lead to the elevation of TRIF polyubiquitination.

\section{DISCUSSION}

As one of transcriptional regulators, members the NF- $\kappa \mathrm{B} / \mathrm{I} \kappa \mathrm{B}$ family can promote the expression of over 100 target genes, the majority of which involved in the host immune responses (34). However, excessive inflammation may damage airframe and result in autoimmune diseases. Therefore, to maintain immune balance, the NF- $\kappa \mathrm{B}$ signaling pathway is tightly regulated. For instance, WWP2 (23), NLRX1 (6), and TRIM38 (35) can negatively regulate TLR-induced signaling pathway by targeting different gene for degradation. In this study, we found that IRF3 negatively regulates TRIF-mediated NF- $\kappa \mathrm{B}$ signaling pathway by targeting TRIF for degradation in fish. Overexpression or knockdown of IRF3 could inhibit TRIF-mediated NF- $\kappa \mathrm{B}$ signaling pathway, whereas its knockdown has an opposite effect. Mechanistically, IRF3 promotes TRIF degradation through the ubiquitinproteasome pathway. However, direct interaction between the IRF3 and TRIF was not observed through co-immunoprecipitation (data not shown). Therefore, our working hypothesis is that the IRF3 would recruit an E3 ligase or enhance its activity and then induced the TRIF ubiquitination. Furthermore, we find that the IAD domain of IRF3 is crucial to proteasomal degradation of TRIF. Therefore, we speculate that IRF3 inhibits TRIF expression, thereby decreasing the expression of downstream cytokines.

In innate and adaptive immune responses, IFN gene expression is regulated by IRF family proteins $(36,37)$. Currently, 11 members of the IRF family have been found in fish (38). Most IRFs can positively regulate IFN expression (39) and are divided into the positive regulators (IRF1, IRF3, IRF4, IRF5, IRF6, IRF7, IRF8, IRF9, and IRF11) and negative regulators (IRF2 and IRF10) according to the capacity of IFN regulation (40-45). However, in addition to regulating the expression of IFN, increasing evidence suggests that IRFs are also involved in the regulation of many innate signaling pathways. For instances, IRF4 (24) and IRF5 (25) affect downstream of the TLR-MyD88 signaling pathways; and IRF8 interacts with TRAF6 and regulate the production of inflammatory mediators (46). In addition, IRF3 and IRF7 can interact with MyD88 and regulate the IRF-induced IFN response in Atlantic salmon (47). IRF family members have been found and reported that they had 
different function in different signaling pathway. However, in our study, we first found that IRF3 could negatively regulate the TRIFmediated NF- $\mathrm{\kappa B}$ signaling pathway. This feedback regulation mechanism ensures the relative balance of immunity.

Most IRF proteins contain an N-terminal DBD and a C-terminal IAD. IRF3 has another domain (SRD) at the C-terminus apart from DBD and IAD. The DBD is crucial for interaction of IRF with IFN stimulatory response elements $(39,48)$. A previous study indicates that the DBD is the core unit that interacts with the promoter of target genes engaged in the IFN pathway. Moreover, except for IRF1 and IRF2, the IADs form a homodimer that recruits other transcription factors (37) and are involved in protein-protein interaction with other IRFs or related molecules (44). In this study, we found that the IAD domain of IRF3 affected TRIF degradation, and the inhibitory effect was weakened, even eliminated when IAD is deleted. This result indeed confirms that the IAD domain of IRFs interacts with related molecules.

Most IRFs found in fish are homologous with those found in mammals, but several differences still exist between fish and mammals. In particular, IRF4a, IRF4b, and IRF10 are found in fish, but not in mammals, suggesting that conservation and evolution coexist from lower vertebrates to mammals. For instances, IRF4 functions as a negative regulator of IFN expression through competing with IRF5 to interact with MyD88 in mammals (24). By contrast, IRF4 promotes the transcription of IFN as a positive regulator in fish (40). Moreover, IRF6 has no effect on IFN

\section{REFERENCES}

1. Akira S, Uematsu S, Takeuchi O. Pathogen recognition and innate immunity. Cell (2006) 124:783-801. doi:10.1016/j.cell.2006.02.015

2. Rehwinkel J, e Sousa CR. RIGorous detection: exposing virus through RNA sensing. Science (2010) 327:284-6. doi:10.1126/science.1185068

3. Takeuchi O, Akira S. Pattern recognition receptors and inflammation. Cell (2010) 140:805-20. doi:10.1016/j.cell.2010.01.022

4. Ting JPY, Duncan JA, Lei Y. How the noninflammasome NLRs function in the innate immune system. Science (2010) 327:286-90. doi:10.1126/science. 1184004

5. Kingeter LM, Lin X. C-type lectin receptor-induced NF- $\mathrm{KB}$ activation in innate immune and inflammatory responses. Cell Mol Immunol (2012) 9: 105-12. doi:10.1038/cmi.2011.58

6. Xia X, Cui J, Wang HY, Zhu L, Matsueda S, Wang Q, et al. NLRX1 negatively regulates TLR-induced NF-KB signaling by targeting TRAF6 and IKK. Immunity (2011) 34:843-53. doi:10.1016/j.immuni.2011.02.022

7. Karin M, Lawrence T, Nizet V. Innate immunity gone awry: linking microbial infections to chronic inflammation and cancer. Cell (2006) 124:823-35. doi:10.1016/j.cell.2006.02.016

8. Liew FY, Xu D, Brint EK, O’Neill LA. Negative regulation of toll-like receptormediated immune responses. Nat Rev Immunol (2005) 5:446-58. doi:10.1038/ nri1630

9. Kawai T, Akira S. Innate immune recognition of viral infection. Nat Immunol (2006) 7:131-7. doi:10.1038/ni1303

10. Zhang YB, Gui JF. Molecular regulation of interferon antiviral response in fish. Dev Comp Immunol (2012) 38:193-202. doi:10.1016/j.dci.2012.06.003

11. Zou J, Secombes CJ. Teleost fish interferons and their role in immunity. Dev Comp Immunol (2011) 35:1376-87. doi:10.1016/j.dci.2011.07.001

12. Moore CB, Bergstralh DT, Duncan JA, Lei Y, Morrison TE, Zimmermann AG, et al. NLRX1 is a regulator of mitochondrial antiviral immunity. Nature (2008) 451:573-7. doi:10.1038/nature06501

13. Zhao Y, Sun X, Nie X, Sun L, Tang TS, Chen D, et al. COX5B regulates MAVSmediated antiviral signaling through interaction with ATG5 and repressing ROS production. PLoS Pathog (2012) 8:e1003086. doi:10.1371/journal.ppat.1003086 production in mammals, while it can significantly activated IFN transcription in fish (40). IRF3 is a typical ISG that is upregulated after stimulation with IFN or a virus. However, in this study, we discovered that IRF3 could negatively regulate TRIF-mediated NF- $\kappa \mathrm{B}$ signaling pathway by targeting TRIF for degradation. In addition, to detect whether the similarly role in mammal, we found that IRF3 of human also may promote the degradation of TRIF (date not shown). This result suggests that the function of same gene is conserved from lower vertebrate to mammals.

The current study has identified that IRF3 as a novel negative regulator inhibits TRIF-mediated NF- $\mathrm{BB}$ signaling pathway by targeting TRIF for degradation through the ubiquitin-proteasomal degradation pathway in fish. These dattum enrich content of TLR signaling pathway in teleosts, and provide new insights into the regulatory mechanism in mammals.

\section{AUTHOR CONTRIBUTIONS}

Conceived and designed the experiments: TX. Performed the experiments: XZ, RH, XY, and TX. Analyzed the data: XZ and TX. Wrote the paper: XZ and TX.

\section{ACKNOWLEDGMENTS}

This study was supported by National Natural Science Foundation of China (31672682).

14. Yu Y, Hayward GS. The ubiquitin E3 ligase RAUL negatively regulates type interferon through ubiquitination of the transcription factors IRF7 and IRF3. Immunity (2010) 33:863-77. doi:10.1016/j.immuni.2010.11.027

15. O'Neill LAJ, Bowie AG. The family of five: TIR-domain-containing adaptors in toll-like receptor signalling. Nat Rev Immunol (2007) 7:353-64. doi:10.1038/ nri2079

16. Kondo T, Kawai T, Akira S. Dissecting negative regulation of toll-like receptor signaling. Trends Immunol (2012) 33:449-58. doi:10.1016/j.it.2012.05.002

17. Zhong B, Zhang L, Lei C, Li Y, Mao AP, Yang Y, et al. The ubiquitin ligase RNF5 regulates antiviral responses by mediating degradation of the adaptor protein MITA. Immunity (2009) 30:397-407. doi:10.1016/j.immuni.2009.01.008

18. Medzhitov R. Toll-like receptors and innate immunity. Nat Rev Immunol (2001) 1:135-45. doi:10.1038/35100529

19. Beutler B, Jiang Z, Georgel P, Crozat K, Croker B, Rutschmann S, et al. Genetic analysis of host resistance: toll-like receptor signaling and immunity at large. Annu Rev Immunol (2006) 24:353-89. doi:10.1146/annurev.immunol.24. 021605.090552

20. Kawai T, Akira S. The role of pattern-recognition receptors in innate immunity: update on toll-like receptors. Nat Immunol (2010) 11:373-84. doi:10.1038/ni.1863

21. Ahmed S, Maratha A, Butt AQ, Shevlin E, Miggin SM. TRIF-mediated TLR3 and TLR4 signaling is negatively regulated by ADAM15. J Immunol (2013) 190:2217-28. doi:10.4049/jimmunol.1201630

22. Xue Q, Zhou Z, Lei X, Liu X, He B, Wang J, et al. TRIM38 negatively regulates TLR3-mediated IFN- $\beta$ signaling by targeting TRIF for degradation. PLoS One (2012) 7:e46825. doi:10.1371/journal.pone.0046825

23. Yang Y, Liao B, Wang S, Yan B, Jin Y, Shu HB, et al. E3 ligase WWP2 negatively regulates TLR3-mediated innate immune response by targeting TRIF for ubiquitination and degradation. Proc Natl Acad Sci US A (2013) 110:5115-20. doi:10.1073/pnas.1220271110

24. Negishi H, Ohba Y, Yanai H, Takaoka A, Honma K, Yui K, et al. Negative regulation of toll-like-receptor signaling by IRF-4. Proc Natl Acad Sci U S A (2005) 102:15989-94. doi:10.1073/pnas.0508327102

25. Takaoka A, Yanai H, Kondo S, Duncan G. Integral role of IRF-5 in the gene induction programme activated by toll-like receptors. Nature (2005) 434:243. doi:10.1038/nature03308 
26. Xu T, Xu G, Che R, Wang R, Wang Y, Li J, et al. The genome of the miiuy croaker reveals well-developed innate immune and sensory systems. Sci Rep (2016) 6:21902. doi:10.1038/srep21902

27. Wang Y, Bi X, Chu Q, Xu T. Discovery of toll-like receptor 13 exists in the teleost fish: miiuy croaker (Perciformes, Sciaenidae). Dev Comp Immunol (2016) 61:25-33. doi:10.1016/j.dci.2016.03.005

28. Wang Y, Li J, Han J, Shu C, Xu T. Identification and characteristic analysis of TLR28: a novel member of the TLR1 family in teleost. Dev Comp Immunol (2016) 62:102-7. doi:10.1016/j.dci.2016.05.001

29. Zhao X, Chu Q, Cui J, Huo R, Xu T. IRF9 as a negative regulator involved in TRIF-mediated NF- $\kappa \mathrm{B}$ pathway in a teleost fish, Miichthys miiuy. Mol Immunol (2017) 85:123-9. doi:10.1016/j.molimm.2017.02.009

30. Chu Q, Sun Y, Cui J, Xu T. MicroRNA-3570 modulates the NF- $\kappa B$ pathway in teleost fish by targeting MyD88. J Immunol (2017) 198:3274-82. doi:10.4049/ jimmunol.1602064

31. Chu Q, Sun Y, Cui J, Xu T. Inducible microRNA-214 contributes to the suppression of NF- $\kappa \mathrm{B}$-mediated inflammatory response via targeting myd 88 gene in fish. J Biol Chem (2017) 292:5282-90. doi:10.1074/jbc.M117.777078

32. Chu Q, Sun Y, Bi D, Cui J, Xu T. Up-regulated of miR-8159-5p and miR-217-5p by LPS stimulation negatively co-regulate TLR1 in miluy croaker. Dev Comp Immunol (2017) 67:117-25. doi:10.1016/j.dci.2016.11.004

33. Livak KJ, Schmittgen TD. Analysis of relative gene expression data using real-time quantitative PCR and the $2^{-\Delta \Delta \mathrm{CT}}$ method. Methods (2001) 25:402-8. doi:10.1006/meth.2001.1262

34. Ghosh S, May MJ, Kopp EB. NF-кB and Rel proteins: evolutionarily conserved mediators of immune responses. Annu Rev Immunol (1998) 16:225-60. doi:10.1146/annurev.immunol.16.1.225

35. Zhao W, Wang L, Zhang M, Yuan C, Gao C. E3 ubiquitin ligase tripartite motif 38 negatively regulates TLR-mediated immune responses by proteasomal degradation of TNF receptor-associated factor 6 in macrophages. J Immunol (2012) 188:2567-74. doi:10.4049/jimmunol.1103255

36. Stark GR. How cells respond to interferons revisited: from early history to current complexity. Cytokine Growth Factor Rev (2007) 18:419-23. doi:10.1016/j. cytogfr.2007.06.013

37. Ozato K, Tailor P, Kubota T. The interferon regulatory factor family in host defense: mechanism of action. J Biol Chem (2007) 282:20065-9. doi:10.1074/ jbc.R700003200

38. Huang B, Qi ZT, Xu Z, Nie P. Global characterization of interferon regulatory factor (IRF) genes in vertebrates: glimpse of the diversification in evolution. BMC Immunol (2010) 11:22. doi:10.1186/1471-2172-11-22

39. Honda K, Taniguchi T. IRFs: master regulators of signalling by toll-like receptors and cytosolic pattern-recognition receptors. Nat Rev Immunol (2006) 6:644. doi: $10.1038 /$ nri1900
40. Li S, Lu LF, Wang ZX, Chen DD, Zhang YA. Fish IRF6 is a positive regulator of IFN expression and involved in both of the MyD88 and TBK1 pathways. Fish Shellfish Immunol (2016) 57:262-8. doi:10.1016/j.fsi.2016.08.059

41. Feng H, Zhang YB, Zhang QM, Li Z, Zhang QY, Gui JF. Zebrafish IRF1 regulates IFN antiviral response through binding to IFN 1 1 and IFN $\phi 3$ promoters downstream of MyD88 signaling. J Immunol (2015) 194:1225-38. doi:10.4049/jimmunol.1402415

42. Holland JW, Bird S, Williamson B, Woudstra C, Mustafa A, Wang T, et al. Molecular characterization of IRF3 and IRF7 in rainbow trout, Oncorhynchus mykiss: functional analysis and transcriptional modulation. Mol Immunol (2008) 46:269-85. doi:10.1016/j.molimm.2008.08.265

43. Shi J, Zhang YB, Zhang JS, Gui JF. Expression regulation of zebrafish interferon regulatory factor 9 by promoter analysis. Dev Comp Immunol (2013) 41:534-43. doi:10.1016/j.dci.2013.07.017

44. Li S, Lu LF, Feng H, Wu N, Chen DD, Zhang YB, et al. IFN regulatory factor 10 is a negative regulator of the IFN responses in fish. J Immunol (2014) 193:1100-9. doi:10.4049/jimmunol.1400253

45. Gu M, Lin G, Lai Q, Zhong B, Liu Y, Mi Y, et al. Ctenopharyngodon idella IRF2 plays an antagonistic role to IRF1 in transcriptional regulation of IFN and ISG genes. Dev Comp Immunol (2015) 49:103-12. doi:10.1016/j.dci.2014. 11.014

46. Zhao J, Kong HJ, Li H, Huang B, Yang M, Zhu C, et al. IRF-8/interferon (IFN) consensus sequence-binding protein is involved in toll-like receptor (TLR) signaling and contributes to the cross-talk between TLR and IFN- $\gamma$ signaling pathways. J Biol Chem (2006) 281:10073-80. doi:10.1074/jbc.M507788200

47. Iliev DB, Sobhkhez M, Fremmerlid K, Jørgensen JB. MyD88 interacts with interferon regulatory factor (IRF) 3 and IRF7 in Atlantic Salmon (Salmo salar) transgenic SsMyD88 modulates the IRF-Induced type I interferon response and accumulates in aggresomes. J Biol Chem (2011) 286:42715-24. doi:10.1074/jbc.M111.293969

48. Paun A, Pitha PM. The IRF family, revisited. Biochimie (2007) 89:744-53. doi:10.1016/j.biochi.2007.01.014

Conflict of Interest Statement: The authors declare that the research was conducted in the absence of any commercial or financial relationships that could be construed as a potential conflict of interest.

Copyright (c) 2018 Zhao, Huo, Yan and Xu. This is an open-access article distributed under the terms of the Creative Commons Attribution License (CC BY). The use, distribution or reproduction in other forums is permitted, provided the original author(s) and the copyright owner are credited and that the original publication in this journal is cited, in accordance with accepted academic practice. No use, distribution or reproduction is permitted which does not comply with these terms. 